Proceedings of the Edinburgh Mathematical Society (2008) 51, 315-335 (C)

DOI:10.1017/S0013091506000319 Printed in the United Kingdom

\title{
GREEN'S FUNCTIONS AND REGULARIZED TRACES OF STURM-LIOUVILLE OPERATORS ON GRAPHS
}

\author{
SONJA CURRIE AND BRUCE A. WATSON \\ School of Mathematics, University of the Witwatersrand, Private Bag 3, Wits 2050, \\ South Africa (sonja.currie@wits.ac.za; b.alastair.watson@gmail.com)
}

(Received 2 March 2006)

\begin{abstract}
Asymptotic approximations to the Green's functions of Sturm-Liouville boundary-value problems on graphs are obtained. These approximations are used to study the regularized traces of the differential operators associated with these boundary-value problems. Various inverse spectral problems for Sturm-Liouville boundary-value problems on graphs resembling those considered in Halberg and Kramer's 'A generalization of the trace concept' (Duke Mathematics Journal 27 (1960), 607-617), for Sturm-Liouville problems, and Pielichowski's 'An inverse spectral problem for linear elliptic differential operators' (Universitatis Iagellonicae Acta Mathematica 27 (1988), 239-246), for elliptic boundary-value problems, are solved.
\end{abstract}

Keywords: differential operators; spectrum; graphs

2000 Mathematics subject classification: Primary 47E05; 34L05; 34B45

\section{Introduction}

Regularized traces of particular ordinary differential operators have been considered by Halberg and Kramer [25], Gesztesy et al. [20], Gesztesy and Simon [19], Gilbert and Kramer $[\mathbf{2 1}, \mathbf{2 2}]$ and Javrjan $[\mathbf{2 8}]$. The two main theorems of Halberg and Kramer $[\mathbf{2 5}$, Theorems 1 and 2] form the foundations for our work; see $\S 2$ for more details. Halberg and Kramer apply these theorems to the Sturm-Liouville equation

$$
l y=\lambda y,
$$

where

$$
l y:=-\frac{\mathrm{d}^{2} y}{\mathrm{~d} x^{2}}+q(x) y
$$

on a compact interval with general Lagrange self-adjoint boundary conditions. Javrjan [28] extended this approach to singular Sturm-Liouville equations, while Gilbert and Kramer treat various higher-order problems in $[\mathbf{2 1}, \mathbf{2 2}]$.

Belokolos et al. $[\mathbf{4}]$, Carlson [10] and Clark et al. $[\mathbf{1 2}, \mathbf{1 3}]$ considered regularized traces in the context of differential systems.

Bochnek [5, 6] and Pielichowski $[\mathbf{3 1}, \mathbf{3 2}]$ used the regularized trace in the setting of elliptic partial differential operators to deduce various inverse spectral results. Combining 
the approaches of $[\mathbf{2 5}]$ and $[\mathbf{3 1}, \mathbf{3 2}]$ we are able to prove various inverse spectral theorems for Sturm-Liouville operators on compact graphs.

We note that the regularized trace of an operator considered here and in $[\mathbf{1 0}, \mathbf{1 2}, \mathbf{2 1}$, $\mathbf{2 2}, \mathbf{2 8}, \mathbf{3 1}, \mathbf{3 2}]$ is as follows. If, in a Hilbert space, $A$ is a lower-semi-bounded self-adjoint operator and $V$ is a bounded self-adjoint operator such that both $A$ and $A+V$ have only discrete spectrum, say $\mu_{0} \leqslant \mu_{1} \leqslant \cdots$ and $\lambda_{0} \leqslant \lambda_{1} \leqslant \cdots$, respectively (where eigenvalues are repeated according to multiplicity), with $\sum\left(\lambda_{j}-\mu_{j}\right)$ convergent, then this summation is called the regularized trace of $A+V$ with respect to $A$.

Boundary-value problems on graphs have been studied by many authors, see the special issue of Waves and Random Media (Volume 12), dedicated to differential operators on graphs, for a review of some of the activity in this area. We refer the reader to [8] for an in-depth look at self-adjointness of Sturm-Liouville operators on graphs. Oscillation theory for Sturm-Liouville problems on graphs was explored in [36]. The eigenvalue asymptotics and variational formulation of the boundary-value problem used here are taken from [16] but also appear, under slightly different assumptions, in [2], of which we were unaware at the time of writing $[\mathbf{1 6}]$.

As regards inverse spectral problems on graphs, the recent paper of Yurko [40] should be noted for showing the dependence of the potential on (his) Weyl function for SturmLiouville operators on a tree. The reader should also note $[\mathbf{7 , 9}, \mathbf{2 4}, \mathbf{2 9}, \mathbf{3 3}-\mathbf{3 5}, \mathbf{4 1}]$ for their consideration of other inverse spectral problems on graphs. The related but distinct inverse spectral problem for the matrix Sturm-Liouville operators with Dirichlet boundary conditions has been considered by many authors: see the bibliography in [12] for an extensive list. Closer to the problem at hand is the inverse spectral problem for the matrix Hill equation, studied in $[\mathbf{1 1}, \mathbf{1 7}]$ and, notably via regularized traces, by Carlson in [10]. For some aspects of the explicit connections between Sturmian systems and Sturm-Liouville operators on graphs we refer the reader to $[\mathbf{1 5}, \mathbf{3 8}]$.

In this paper we consider a directed graph, $G$, with finitely many edges, $e_{i}, i=1, \ldots, K$, each of finite length $l_{i}$. The edge $e_{i}$ is identified with the interval $\left[0, l_{i}\right]$. Here 0 is associated with the initial point of $e_{i}$ and $l_{i}$ with the terminal point of $e_{i}$. The focus of our study is the Sturm-Liouville equation (1.1) on the graph $G$, where (1.1) becomes a shorthand for the system of equations

$$
-\frac{\mathrm{d}^{2} y_{i}}{\mathrm{~d} x^{2}}+q_{i}(x) y_{i}=\lambda y_{i}, \quad x \in\left[0, l_{i}\right], i=1, \ldots, K,
$$

where $q_{i}$ and $y_{i}$ denote $\left.q\right|_{e_{i}}$ and $\left.y\right|_{e_{i}}$. Here each $q_{i}$ is a real-valued $\mathcal{L}^{\infty}\left[0, l_{i}\right]$ function. It should be noted that by $\partial G$ we mean the set of nodes of $G$ and by $G^{\circ}$ the interior of $G$, i.e. $G^{\circ}:=G \backslash \partial G$.

The boundary conditions at the node $\nu$ are specified in terms of the values of $y$ and $y^{\prime}$ at $\nu$ on each of the incident edges. In particular, if the edges that start at $\nu$ are $e_{i}$, $i \in \Lambda_{s}(\nu)$, and the edges that end at $\nu$ are $e_{i}, i \in \Lambda_{e}(\nu)$, then the boundary conditions at $\nu$ can be expressed as

$$
\sum_{j \in \Lambda_{s}(\nu)}\left[\alpha_{i j} y_{j}+\beta_{i j} y_{j}^{\prime}\right](0)+\sum_{j \in \Lambda_{e}(\nu)}\left[\gamma_{i j} y_{j}+\delta_{i j} y^{\prime}{ }_{j}\right]\left(l_{j}\right)=0, \quad i=1, \ldots, N(\nu),
$$


where $N(\nu)$ is the number of linearly independent boundary conditions at node $\nu$. The boundary conditions at each node are assumed to be formally self-adjoint, i.e. the system is Lagrange self-adjoint in the sense that

$$
(l f, g)-(f, l g)=0 \quad \text { for all } f, g \in \mathcal{C}^{2}(G) \text { obeying (1.4). }
$$

A consequence of the formal self-adjointness is that (1.4) imposes $2 K$ linearly independent conditions, i.e. $\sum_{\nu} N(\nu)=2 K$ (see $[\mathbf{8}, \mathbf{3 0}]$ for more details).

After a rescaling of the edges to length 1, the boundary-value problem (1.3), (1.4) is equivalent to a weighted Sturm-Liouville system on $[0,1]$ (see $[\mathbf{1 5}, \mathbf{3 8}]$ ). Hence properties such as self-adjointness, lower semi-boundedness and compactness of the resolvent follow from standard Sturmian systems theory (see $[\mathbf{3}, \mathbf{3 0}, \mathbf{3 7}, \mathbf{3 9}]$ ). In addition, we require the boundary conditions to be of co-normal type (see Definition 5.2) in order to ensure a suitable variational formulation of the boundary-value problem.

The boundary conditions at a node, $\nu$, are said to be of Kirchhoff type if they are of the form

$$
\begin{gathered}
y_{i}(0)=y_{j}(0)=y_{r}\left(l_{r}\right)=y_{s}\left(l_{s}\right) \quad \text { for all } i, j \in \Lambda_{s}(\nu), r, s \in \Lambda_{e}(\nu), \\
\sum_{j \in \Lambda_{e}(\nu)} y^{\prime}{ }_{j}\left(l_{j}\right)-\sum_{j \in \Lambda_{s}(\nu)} y^{\prime}{ }_{j}(0)=0 .
\end{gathered}
$$

We say that the node $\nu$ has boundary conditions of Neumann type if the boundary conditions are of Kirchhoff type and $\# \Lambda_{s}(\nu)+\# \Lambda_{e}(\nu)=1$.

The boundary conditions at $\nu$ are of Dirichlet type if they can be expressed as

$$
\begin{aligned}
y_{i}(0)=0 & \text { for all } i \in \Lambda_{s}(\nu), \\
y_{j}\left(l_{j}\right)=0 & \text { for all } j \in \Lambda_{e}(\nu) .
\end{aligned}
$$

It is easily verified that Dirichlet and Kirchhoff boundary conditions are of co-normal type.

In $\S 6$ we prove our main theorem.

Theorem 1.1. Consider the boundary-value problem on the graph $G$ consisting of (1.3) and boundary conditions that are of Kirchhoff type at each node $\nu$ of G. Let $\tilde{A}$ be the operator generated in $\mathcal{L}^{2}(G)$ from this boundary-value problem with $q \in \mathcal{C}^{2}(G)$, and let $A$ be operator generated from this problem but with $q=0$. If $\lambda_{0}=\mu_{0}$ and $\sum\left(\lambda_{n}-\mu_{n}\right)$ converges, where $\mu_{0}, \mu_{1}, \ldots$ and $\lambda_{0}, \lambda_{1}, \ldots$ are the eigenvalues of $\tilde{A}$ and $A$, respectively, listed in increasing order and repeated according to multiplicity, then $q$ is identically 0 on $G$.

\section{Preliminaries}

A compact self-adjoint operator $C$ on a Hilbert space is said to be of trace class or 'nuclear' if the sum of its eigenvalues, i.e. its trace, denoted $\operatorname{tr}(C)$ (with repetition according to multiplicity), is absolutely convergent (see [23, pp. 95-106]). 
Let $T$, a self-adjoint operator on Hilbert space $H$ with domain $\mathcal{D}(T)$, be semi-bounded from below and let $(T-\mu I)^{-1}$ be of trace class for $\mu \leqslant M$, for some $M<0$. Denote the eigenvalues of $T$ by $\mu_{0} \leqslant \mu_{1} \leqslant \cdots$, where eigenvalues are repeated according to multiplicity. Associate with this sequence of eigenvalues a corresponding complete orthonormal sequence of eigenfunctions $\varphi_{0}, \varphi_{1}, \ldots$ In this context, Halberg and Kramer [25, Theorems 1 and 2] prove the following theorem, on which this paper relies.

Theorem 2.1 (Halberg and Kramer). Let $V$ be a bounded operator defined on $\mathcal{D}(T)$ such that the operator $T+V$ has a denumerable sequence of real eigenvalues $\lambda_{0} \leqslant \lambda_{1} \leqslant \cdots$ having the property that $\sum_{n=0}^{\infty}\left(\lambda_{n}-\mu_{n}\right)$ is convergent. Then $(T-\mu I)^{-1} V(T-\mu I)^{-1}$ is of trace class for $\mu \leqslant M$, and

$$
\sum_{n=0}^{\infty}\left(\lambda_{n}-\mu_{n}\right)=\lim _{\mu \rightarrow-\infty} \mu^{2} \operatorname{tr}\left[(T-\mu I)^{-1} V(T-\mu I)^{-1}\right] .
$$

If, in addition, $\sum_{n=0}^{\infty}\left(V \varphi_{n}, \varphi_{n}\right)$ is convergent, then

$$
\sum_{n=0}^{\infty}\left(\lambda_{n}-\mu_{n}\right)=\sum_{n=0}^{\infty}\left(V \varphi_{n}, \varphi_{n}\right)
$$

It should be noted that in order to obtain (2.1) from the above theorem, we need to verify the following conditions on the self-adjoint operator $T$ and the bounded operator $V$ in $H$ :

(a) $T$ is lower semi-bounded;

(b) there exists $M<0$ such that $(T-\mu I)^{-1}$ is of trace class for $\mu \leqslant M$;

(c) $T+V$ has a denumerable sequence of (real) eigenvalues;

(d) $\sum_{n=0}^{\infty}\left(\lambda_{n}-\mu_{n}\right)$ is convergent.

Here (d) will hold by assumption. In order to obtain (2.2) we, in addition, need $V$ to be a trace class operator in $\mathcal{L}^{2}(G)$.

The following function spaces provide the setting for our work. The first three are Hilbert spaces when given Sobolev norms:

$$
\begin{aligned}
\mathcal{L}^{2}(G) & :=\bigoplus_{i=1}^{K} \mathcal{L}^{2}\left(0, l_{i}\right), \\
\mathcal{H}_{\circ}^{m}(G) & :=\bigoplus_{i=1}^{K} \mathcal{H}_{\circ}^{m}\left(0, l_{i}\right), \quad m=0,1,2, \ldots, \\
\mathcal{H}^{m}(G) & :=\bigoplus_{i=1}^{K} \mathcal{H}^{m}\left(0, l_{i}\right), \quad m=0,1,2, \ldots,
\end{aligned}
$$




$$
\begin{aligned}
& \mathcal{C}^{\omega}(G):=\bigoplus_{i=1}^{K} \mathcal{C}^{\omega}\left(\left[0, l_{i}\right]\right), \quad \omega=\infty, 0,1,2, \ldots, \\
& \mathcal{C}_{\circ}^{\omega}(G):=\bigoplus_{i=1}^{K} \mathcal{C}_{\circ}^{\omega}\left(0, l_{i}\right), \quad \omega=\infty, 0,1,2, \ldots
\end{aligned}
$$

The inner product on $\mathcal{H}^{m}(G)$ and $\mathcal{H}_{\circ}^{m}(G)$, denoted $(\cdot, \cdot)_{m}$, is defined by

$$
(f, g)_{m}:=\left.\left.\sum_{i=1}^{K} \sum_{j=0}^{m} \int_{0}^{l_{i}} f\right|_{e_{i}} ^{(j)} \bar{g}\right|_{e_{i}} ^{(j)} \mathrm{d} t=: \sum_{j=0}^{m} \int_{G} f^{(j)} \bar{g}^{(j)} \mathrm{d} t .
$$

Let $\|f\|_{m}^{2}:=(f, f)_{m}$. We will write $(f, g)$ for $(f, g)_{0}$ and $\|f\|$ for $\|f\|_{0}$.

The boundary-value problem (1.3), (1.4) on $G$ can be formulated as an operator eigenvalue problem in $\mathcal{L}^{2}(G)$ by setting

$$
A f:=-f^{\prime \prime}+q f
$$

with domain

$$
\mathcal{D}(A)=\left\{f \mid f, f^{\prime} \in A C, A f \in \mathcal{L}^{2}(G), f \text { obeying (1.4) }\right\}
$$

(see $[\mathbf{2}, \mathbf{8}, \mathbf{1 6}]$, and for systems formulations of $(1.3),(1.4)$ see $[\mathbf{1 5}, \mathbf{3 8}])$.

Note that since $q \in \mathcal{L}^{\infty}(G)$ an equivalent definition of $\mathcal{D}(A)$ is

$$
\mathcal{D}(A)=\left\{f \in \mathcal{H}^{2}(G) \mid f \text { obeys }(1.4)\right\},
$$

which is clearly independent of $q$. The operator $A$ thus defined is a lower-semi-bounded self-adjoint operator with compact resolvent in $\mathcal{L}^{2}(G)$ (see $[\mathbf{2 7}, \mathbf{3 0}, \mathbf{3 9}]$ or $[\mathbf{3 7}$, Chapter 7]).

Set $A_{0}$ to be the principal part of $A$ and let $V_{p}$, for each real-valued $p \in \mathcal{L}^{\infty}(G)$, denote the multiplier operator

$$
V_{p} f=p f \quad \text { for all } f \in \mathcal{L}^{2}(G) .
$$

Observe that $A=A_{0}+V_{q}$ and that $V_{q}$ is a bounded self-adjoint operator in $\mathcal{L}^{2}(G)$ for each real-valued $q \in \mathcal{L}^{\infty}(G)$.

\section{Green's function}

Formulating (1.3), (1.4) as a second-order system with separated boundary conditions (see [15]) gives directly that the boundary-value problem has a Green's function and that the Green's operator is a compact operator. Denote the iterated Green's function by $g^{k}(x, y, \lambda), k \in \mathbb{N}$, i.e. the kernel of the operator $G_{\lambda}^{k}:=(A-\lambda I)^{-k}$. We give an analogue of $[\mathbf{1 8}, \S 3]$ for iterated Green's functions on a graph: Lemma 3.1. In particular, for $\rho>0$ large,

$$
G_{-\rho^{2}}^{k} f(y)=\int_{G} g^{k}\left(y, x,-\rho^{2}\right) f(x) \mathrm{d} x \quad \text { for all } f \in \mathcal{L}^{2}(G),
$$


and

$$
\begin{aligned}
\left(l+\rho^{2}\right)^{k} G_{-\rho^{2}}^{k} f=f & \text { for all } f \in \mathcal{L}^{2}(G), \\
G_{-\rho^{2}}\left(l+\rho^{2}\right) f=f & \text { for all } f \in \mathcal{D}(A) .
\end{aligned}
$$

For $x \neq y$,

$$
\left(l_{x}+\rho^{2}\right) G_{-\rho^{2}}\left(x, y,-\rho^{2}\right)=0
$$

where $l_{x}$ denotes $l$ operating with respect to the variable $x$, with $y$ held constant. As the boundary-value problem is self-adjoint,

$$
G_{-\rho^{2}}\left(y, x,-\rho^{2}\right)=G_{-\rho^{2}}\left(x, y,-\rho^{2}\right),
$$

for $\rho \in \mathbb{R}$.

Let $\mathbb{A}$ denote the operator $A$ generated when (1.4) is replaced by Dirichlet boundary conditions at all nodes, and denote by $\Gamma$ and $\gamma$ the Green's operator and function corresponding to $\mathbb{A}$.

Lemma 3.1. Let $k \in \mathbb{N}, q \in \mathcal{C}^{2(k-1)}(G)$ be real valued, and let $U \subset G$ be open with $\bar{U} \subset G^{\circ}$. If $r=\frac{1}{3} \operatorname{dist}(\partial G, \bar{U})$, then

$$
\left|g^{k}\left(y, z,-\rho^{2}\right)-\gamma^{k}\left(y, z,-\rho^{2}\right)\right| \leqslant \frac{C(U)}{\rho^{2} \mathrm{e}^{2 r \rho}} \quad \text { for } y, z \in U,
$$

where $C(U)>0$ is independent of $\rho$ and $y, z$.

Proof. The sesquilinear forms generated by $g^{k}\left(x, y,-\rho^{2}\right)$ and $\gamma^{k}\left(x, y,-\rho^{2}\right)$ will, respectively, be denoted by

$$
G_{\rho}^{k}(f, h):=\int_{G} \int_{G} f(x) g^{k}\left(x, y,-\rho^{2}\right) h(y) \mathrm{d} x \mathrm{~d} y,
$$

and

$$
\Gamma_{\rho}^{k}(f, g):=\int_{G} \int_{G} f(x) \gamma^{k}\left(x, y,-\rho^{2}\right) g(y) \mathrm{d} x \mathrm{~d} y,
$$

for $f, h \in \mathcal{L}^{2}(G)$. Now $\lambda_{0}(g, g) \leqslant(A g, g)$ for all $g \in \mathcal{D}(A)$, and consequently $\left(\rho^{2}+\right.$ $\left.\lambda_{0}\right)(g, g) \leqslant\left(\left(A+\rho^{2}\right) g, g\right)$, from which it follows that, for all $\rho^{2}>\lambda_{0}$,

$$
0 \leqslant\left(\lambda_{0}+\rho^{2}\right)\|g\|^{2} \leqslant\left\|\left(A+\rho^{2}\right) g\right\|\|g\| .
$$

Thus

$$
0 \leqslant\left(\lambda_{0}+\rho^{2}\right)\|g\| \leqslant\left\|\left(A+\rho^{2}\right) g\right\| \text { for all } g \in \mathcal{D}(A) .
$$

Let $h \in \mathcal{L}^{2}(G)$ and $g:=G_{\rho} h$, then the above display gives

$$
\left\|G_{\rho} h\right\| \leqslant \frac{\|h\|}{\lambda_{0}+\rho^{2}}
$$


from which it follows immediately that

$$
\left\|G_{\rho}\right\| \leqslant \frac{1}{\lambda_{0}+\rho^{2}} \quad \text { for all } \rho^{2}>\lambda_{0} .
$$

Hence there exists $\kappa>0$ such that, for $\rho>0$ sufficiently large,

$$
\left\|G_{\rho}^{k}\right\| \leqslant \frac{\kappa}{\rho^{2 k}}
$$

and thus, for $k \in \mathbb{N}$ and $\rho>0$ large,

$$
\left|G_{\rho}^{k}(f, h)\right| \leqslant \frac{\kappa}{\rho^{2 k}}\|f\|\|h\| \text { for all } f, h \in \mathcal{L}^{2}(G),
$$

with a similar bound holding for $\Gamma_{\rho}^{k}$. Now let

$$
C_{\rho}^{k}(f, g):=G_{\rho}^{k}(f, g)-\Gamma_{\rho}^{k}(f, g) .
$$

From (3.6) and its analogue for $\Gamma_{\rho}^{k}$, there exists a constant $\kappa_{1}>0$ such that, for large $\rho>0$,

$$
\left\|C_{\rho}^{k}\right\|=\sup _{f, g \in \mathcal{L}^{2}(G) \backslash\{0\}} \frac{\left|C_{\rho}^{k}(f, g)\right|}{\|f\|\|g\|}=\sup _{f, g \in \mathcal{L}^{2}(G) \backslash\{0\}} \frac{\left|G_{\rho}^{k}(f, g)-\Gamma_{\rho}^{k}(f, g)\right|}{\|f\|\|g\|} \leqslant \frac{\kappa_{1}}{\rho^{2 k}} .
$$

Thus $\left\|C_{\rho}^{k}\right\|=O\left(\rho^{-2 k}\right)$.

Since

$$
\mathcal{H}_{\circ}^{2 k}(G) \subset \mathcal{D}(A) \cap \mathcal{D}(\mathbb{A})
$$

it follows that

$$
A^{k} h=\mathbb{A}^{k} h=l^{k} h \quad \text { for all } h \in \mathcal{H}_{\circ}^{2 k}(G) .
$$

Let

$$
b_{\rho}^{k} h:=\left(A+\rho^{2}\right)^{k} h=\left(\mathbb{A}+\rho^{2}\right)^{k} h=\left(l+\rho^{2}\right)^{k} h \quad \text { for all } h \in \mathcal{H}_{\circ}^{2 k}(G) .
$$

From the definitions of $g^{k}\left(x, y,-\rho^{2}\right)$ and $\gamma^{k}\left(x, y,-\rho^{2}\right)$,

$$
G_{\rho}^{k}\left(f, b_{\rho}^{k} h\right)=\left(f, G_{\rho}^{k} b_{\rho}^{k} h\right)=(f, h)=\left(f, \Gamma_{\rho}^{k} b_{\rho}^{k} h\right)=\Gamma_{\rho}^{k}\left(f, b_{\rho}^{k} h\right)
$$

for all $f \in \mathcal{L}^{2}(G)$ and $h \in \mathcal{H}_{\circ}^{2 k}(G)$, and thus, for all such $f$ and $h$,

$$
C_{\rho}^{k}\left(f, b_{\rho}^{k} h\right)=G_{\rho}^{k}\left(f, b_{\rho}^{k} h\right)-\Gamma_{\rho}^{k}\left(f, b_{\rho}^{k} h\right)=0 .
$$

Let $U_{1}, U_{2}$ be open subsets of $G$ with

$$
\bar{U} \subset U_{1} \subset \bar{U}_{1} \subset U_{2} \subset \bar{U}_{2} \subset G^{\circ} .
$$

Let $\varphi \in \mathcal{C}_{\circ}^{\infty}(G)$ with $\left.\varphi\right|_{U_{1}} \equiv 1$ and $\left.\varphi\right|_{G \backslash U_{2}} \equiv 0$. For $y \in U_{1}$ let

$$
p_{\rho}^{k}(y, x):=b_{\rho, x}^{k}\left[\gamma^{k}\left(y, x,-\rho^{2}\right)(1-\varphi(x))\right] \text { for all } x \in G .
$$

Then, for $y \in U_{1}, p_{\rho}^{k}(y, x)$ vanishes everywhere except possibly for $x \in U_{2} \backslash U_{1}$. 
For each $y, y^{*} \in U_{1}$ let

$$
\begin{aligned}
c^{k}\left(y, y^{*},-\rho^{2}\right) & :=C_{\rho}^{k}\left(p_{\rho}^{k}(y, \cdot), p_{\rho}^{k}\left(y^{*}, \cdot\right)\right) \\
& =\int_{G} \int_{G} p_{\rho}^{k}(y, x)\left[g^{k}-\gamma^{k}\right]\left(x, w,-\rho^{2}\right) p_{\rho}^{k}\left(y^{*}, w\right) \mathrm{d} x \mathrm{~d} w
\end{aligned}
$$

and, for each $f, h \in \mathcal{L}^{2}(G)$ with support in $U_{1}$, i.e. $f=\chi_{U_{1}} f$ and $g=\chi_{U_{1}} g$, where $\chi_{U_{1}}$ is the characteristic function of $U_{1}$,

$$
c_{\rho}^{k}(f, h)=\int_{G} \int_{G} f(y) c^{k}\left(y, y^{*},-\rho^{2}\right) h\left(y^{*}\right) \mathrm{d} y \mathrm{~d} y^{*} .
$$

From the continuity of $p_{\rho}^{k}$ and $c_{\rho}^{k}$,

$$
\begin{aligned}
c_{\rho}^{k}(f, h) & =\int_{G} \int_{G} f(y) c^{k}\left(y, y^{*},-\rho^{2}\right) h\left(y^{*}\right) \mathrm{d} y \mathrm{~d} y^{*} \\
& =\int_{G} \int_{G}\left[\int_{G} f(y) p_{\rho}^{k}(y, z) \mathrm{d} y\right]\left[g^{k}-\gamma^{k}\right]\left(x, w,-\rho^{2}\right)\left[\int_{G} p_{\rho}^{k}\left(y^{*}, w\right) h\left(y^{*}\right) \mathrm{d} y^{*}\right] \mathrm{d} w \mathrm{~d} x \\
& =C_{\rho}^{k}\left(\left[\int_{G} f(y) p_{\rho}^{k}(y, \cdot) \mathrm{d} y\right],\left[\int_{G} p_{\rho}^{k}\left(y^{*}, \cdot\right) h\left(y^{*}\right) \mathrm{d} y^{*}\right]\right) \\
& =C_{\rho}^{k}\left(b_{\rho}^{k}\left[(1-\varphi) \Gamma_{\rho}^{k} f\right], b_{\rho}^{k}\left[(1-\varphi) \Gamma_{\rho}^{k} h\right]\right) \\
& =C_{\rho}^{k}\left(f-b_{\rho}^{k} \varphi \Gamma_{\rho}^{k} f, h-b_{\rho}^{k} \varphi \Gamma_{\rho}^{k} h\right),
\end{aligned}
$$

for $f, h \in \mathcal{H}^{2 k}(G)$ with $\operatorname{supp}(f), \operatorname{supp}(h) \subset U_{1}$.

Since $\varphi \Gamma_{\rho}^{k} f, \varphi \Gamma_{\rho}^{k} h \in \mathcal{H}_{\circ}^{2 k}(G)$, by (3.8),

$$
C_{\rho}^{k}\left(f, b_{\rho}^{k} \varphi \Gamma_{\rho}^{k} h\right)=C_{\rho}^{k}\left(b_{\rho}^{k} \varphi \Gamma_{\rho}^{k} f, h\right)=C_{\rho}^{k}\left(b_{\rho}^{k} \varphi \Gamma_{\rho}^{k} f, b_{\rho}^{k} \varphi \Gamma_{\rho}^{k} h\right)=0
$$

and

$$
C_{\rho}^{k}(f, h)=c_{\rho}^{k}(f, h)
$$

Thus

$$
G_{\rho}^{k}(f, h)=\Gamma_{\rho}^{k}(f, h)+c_{\rho}^{k}(f, h),
$$

for $f, h \in \mathcal{H}^{2 k}(G)$ with $\operatorname{supp}(f), \operatorname{supp}(h) \subset U_{1}$. By continuity of the forms $C_{\rho}^{k}, G_{\rho}^{k}$ and $\Gamma_{\rho}^{k},(3.10)$ holds for all $f, h \in \mathcal{L}^{2}(G)$ with supports contained in $U_{1}$. Consequently,

$$
g^{k}\left(z, w,-\rho^{2}\right)=\gamma^{k}\left(z, w,-\rho^{2}\right)+c^{k}\left(z, w,-\rho^{2}\right),
$$

almost everywhere for $z, w \in U_{1}$, and, since $g^{k}\left(z, w,-\rho^{2}\right), \gamma^{k}\left(z, w,-\rho^{2}\right)$ and $c^{k}\left(z, w,-\rho^{2}\right)$ are continuous with respect to $z, w \in G$, (3.11) holds for al2l $z, w \in U_{1}$.

From (3.7) it follows that, for large $\rho>0$ and $y, y^{*} \in U$,

$$
\left|c^{k}\left(y, y^{*},-\rho^{2}\right)\right| \leqslant \frac{\kappa_{2}}{\rho^{2 k}}\left\|p_{\rho}^{k}(y, \cdot)\right\|\left\|p_{\rho}^{k}\left(y^{*}, \cdot\right)\right\| .
$$


Let $y \in U$, then

$$
\left\|p_{\rho}^{k}(y, \cdot)\right\| \leqslant K(\varphi) \sum_{i=0}^{2 k-1} \sup _{x \in U_{2} \backslash U_{1}}\left|\frac{\partial^{i} \gamma^{k}\left(y, x,-\rho^{2}\right)}{\partial x^{i}}\right| .
$$

and, by Corollary 6.3,

$$
\left\|p_{\rho}^{k}(y, \cdot)\right\| \leqslant C(\varphi) \sup _{x \in U_{2} \backslash U_{1}} \mathrm{e}^{-\rho|x-y|} \rho^{k-1}
$$

where $K(\varphi)$ and $C(\varphi)$ depend on $\varphi$ and its derivatives.

Let $r=\operatorname{dist}\left(\bar{U}_{2} \backslash U_{1}, \bar{U}\right)$, then from the above bound and Lemma 6.2 there is a constant $C(\varphi)>0$ such that for $y \in U$

$$
\left\|p_{\rho}(y, \cdot)\right\| \leqslant C(\varphi) \mathrm{e}^{-r \rho} \rho^{k-1}
$$

Hence for all $y, y^{*} \in U$

$$
\left|c^{k}\left(y, y^{*},-\rho^{2}\right)\right| \leqslant \kappa_{2} C^{2}(\varphi) \frac{\mathrm{e}^{-2 r \rho}}{\rho^{2}},
$$

from which the lemma follows directly.

Combining Lemmas 6.2 and 3.1 yields the following corollary.

Corollary 3.2. For $\lambda<-\left|\lambda_{0}\right|$, where $\lambda_{0}$ is the least eigenvalue of (1.3), (1.4), and $q \in \mathcal{C}^{2}(G)$, the iterated Green's function $g^{2}(x, y, \lambda)$ of $(l-\lambda)$ with (1.4) satisfies

$$
\lim _{\rho \rightarrow \infty} \rho^{3} g^{2}\left(x, x,-\rho^{2}\right)=\frac{1}{4} \quad \text { for each } x \in G .
$$

This limit holds uniformly on compact subsets of $G^{\circ}$.

\section{Regularized traces}

In this section we develop the theory of regularized traces for differential operators on graphs. Regularized traces of partial differential operators on regions with smooth boundaries and compact closures were studied in $[\mathbf{5}, \mathbf{2 8}, \mathbf{3 2}]$.

If $A$ and $\tilde{A}$ are lower-semi-bounded self-adjoint semi-simple differential operators with eigenvalues $\lambda_{0} \leqslant \lambda_{1} \leqslant \cdots$ and $\tilde{\lambda}_{0} \leqslant \tilde{\lambda}_{1} \leqslant \cdots$ listed in increasing order and repeated according to multiplicity, then the regularized trace of $A$ with respect to $\tilde{A}$ is $\sum\left(\lambda_{j}-\tilde{\lambda}_{j}\right)$, if this summation converges. This summation is termed the regularized trace of $A$ with respect to $\tilde{A}$ (see $[\mathbf{2 5}]$ ) since neither $A$ nor $\tilde{A}$ need necessarily have finite trace for the regularized trace to be defined.

Lemma 4.1. Let $A$ and $V_{p}, p \in \mathcal{L}^{\infty}(G)$, be as defined in (2.4)-(2.6) and let $\mu_{0}, \mu_{1}, \ldots$ and $\lambda_{0}, \lambda_{1}, \ldots$ be the eigenvalues of $A+V_{p}$ and $A$, respectively, listed in increasing order 
and repeated according to multiplicity. If $\sum_{n=0}^{\infty}\left(\mu_{n}-\lambda_{n}\right)$ is convergent, then $V_{p} A_{\lambda}^{-2}$ is a trace class operator and

$$
\lim _{\lambda \rightarrow-\infty} \lambda^{2} \operatorname{tr}\left(V_{p} A_{\lambda}^{-2}\right)=\sum_{n=0}^{\infty}\left(\mu_{n}-\lambda_{n}\right),
$$

where $A_{\lambda}=A-\lambda I$.

Proof. Assume that $\sum_{n=0}^{\infty}\left(\mu_{n}-\lambda_{n}\right)$ is convergent. Let $\left\{\varphi_{n}\right\}$ be an orthonormal family of eigenfunctions of $A$ corresponding to the eigenvalue sequence $\left\{\lambda_{n}\right\}$. Then

$$
\sum_{n=0}^{\infty}\left|\left(V_{p} A_{\lambda}^{-2} \varphi_{n}, \varphi_{n}\right)\right| \leqslant \sum_{n=0}^{\infty} \frac{\left\|V_{p}\right\|}{\left|\lambda-\lambda_{n}\right|^{2}}<\infty
$$

since there exist constants $0<K_{1}<K_{2}$ such that, for large $n, K_{1} n^{2} \leqslant \lambda_{n} \leqslant K_{2} n^{2}$ (see [15]). Therefore, $V_{p} A_{\lambda}^{-2}$ is a trace class operator. From Theorem 2.1,

$$
\sum_{n=0}^{\infty}\left|\left(A_{\lambda}^{-1} \varphi_{n}, \varphi_{n}\right)\right| \leqslant \sum_{n=0}^{\infty} \frac{1}{\left|\lambda-\lambda_{n}\right|} \leqslant \sum_{n=0}^{\infty} \frac{1}{K_{1} n^{2}-|\lambda|}<\infty .
$$

Now, since $A$ and $A+V_{p}$ both have only discrete spectrum, Theorem 2.1 can be applied to give

$$
\lim _{\lambda \rightarrow-\infty} \lambda^{2} \operatorname{tr}\left(A_{\lambda}^{-1} V_{p} A_{\lambda}^{-1}\right)=\sum_{n=0}^{\infty}\left(\mu_{n}-\lambda_{n}\right) .
$$

The lemma now follows upon noting that, for $\lambda<\lambda_{0}$, the self-adjointness of $A$ gives

$$
\begin{aligned}
\operatorname{tr}\left(A_{\lambda}^{-1} V_{p} A_{\lambda}^{-1}\right) & =\sum_{n=0}^{\infty}\left(A_{\lambda}^{-1} V_{p} A_{\lambda}^{-1} \varphi_{n}, \varphi_{n}\right) \\
& =\sum_{n=0}^{\infty}\left(V_{p} A_{\lambda}^{-1} \varphi_{n}, A_{\lambda}^{-1} \varphi_{n}\right) \\
& =\sum_{n=0}^{\infty} \frac{\left(V_{p} \varphi_{n}, \varphi_{n}\right)}{\left(\lambda_{n}-\lambda\right)^{2}} \\
& =\sum_{n=0}^{\infty}\left(V_{p} A_{\lambda}^{-2} \varphi_{n}, \varphi_{n}\right) .
\end{aligned}
$$

As in [32], the Mercer expansion, together with Corollary 3.2 and Lemma 4.1, shows that the convergence of the regularized trace of $A+V_{p}$ with respect to $A$ implies that the mean value of $p$ is 0 . More precisely we obtain the following theorem.

Theorem 4.2. Let $A, V_{p}, p \in \mathcal{C}^{2}(G)$, be as defined in (2.4)-(2.6) and let $\mu_{0} \leqslant \mu_{1} \leqslant \cdots$ and $\lambda_{0} \leqslant \lambda_{1} \leqslant \cdots$ be the eigenvalues of $A+V_{p}$ and $A$, respectively, repeated according to multiplicity. If $\sum_{n=0}^{\infty}\left(\mu_{n}-\lambda_{n}\right)$ is convergent, then $\int_{G} p(x) \mathrm{d} x=0$. 
Proof. Mercer expansion of $g^{2}(x, y, \lambda)$ gives

$$
g^{2}(x, y, \lambda)=\sum_{n=0}^{\infty} \frac{\varphi_{n}(x) \varphi_{n}(y)}{\left(\lambda_{n}-\lambda\right)^{2}}
$$

where $\left\{\varphi_{n}\right\}$ is an orthonormal sequence of eigenfunctions of $A$ corresponding to the eigenvalue sequence $\left\{\lambda_{n}\right\}$. In particular

$$
g^{2}(x, x, \lambda)=\sum_{n=0}^{\infty} \frac{\varphi_{n}^{2}(x)}{\left(\lambda_{n}-\lambda\right)^{2}},
$$

where the summation

$$
b(x):=\sum_{n=0}^{\infty} \frac{\varphi_{n}^{2}(x)}{\left|\lambda_{n}-\lambda\right|^{2}}
$$

converges both almost everywhere pointwise and in $\mathcal{L}^{1}(G)$ as there exist constants $0<$ $K_{1}<K_{2}$ such that, for large $n, K_{1} n^{2} \leqslant \lambda_{n} \leqslant K_{2} n^{2}$ (see [15]). Thus $b(x) \max |p(x)|$ is an $\mathcal{L}^{1}(G)$-bound for the pointwise-convergent sequence of partial sums

$$
\left\{\sum_{n=0}^{N} \frac{\varphi_{n}^{2}(x) p(x)}{\left(\lambda_{n}-\lambda\right)^{2}}\right\}
$$

Hence Lebesgue's dominated convergence theorem can be applied to give

$$
\int_{G} g^{2}(x, x, \lambda) p(x) \mathrm{d} x=\sum_{n=0}^{\infty} \int_{G} \frac{\varphi_{n}^{2}(x) p(x)}{\left(\lambda_{n}-\lambda\right)^{2}} \mathrm{~d} x=\operatorname{tr}\left(V_{p} A_{\lambda}^{-2}\right) .
$$

Now, as $\sum_{n=0}^{\infty}\left(\mu_{n}-\lambda_{n}\right)$ converges, from Lemma 4.1 we obtain

$$
\lim _{\lambda \rightarrow-\infty} \lambda^{3 / 2} \operatorname{tr}\left(V_{p} A_{\lambda}^{-2}\right)=\lim _{\lambda \rightarrow-\infty} \lambda^{2} \operatorname{tr}\left(V_{p} A_{\lambda}^{-2}\right) \lim _{\lambda \rightarrow-\infty} \lambda^{-1 / 2}=0 .
$$

Hence

$$
0=\lim _{\lambda \rightarrow-\infty} \lambda^{3 / 2} \operatorname{tr}\left(V_{p} A_{\lambda}^{-2}\right)=\lim _{\lambda \rightarrow-\infty} \int_{G} \lambda^{3 / 2} g^{2}(x, x, \lambda) p(x) \mathrm{d} x
$$

The uniformity on compact subsets of $G^{\circ}$ of the limit in Corollary 3.2 allows us to interchange the limit and the summation above, giving

$$
0=\int_{G} \lim _{\lambda \rightarrow-\infty}(-\lambda)^{3 / 2} g^{2}(x, x, \lambda) p(x) \mathrm{d} x=\frac{1}{4} \int_{G} p(x) \mathrm{d} x .
$$

\section{Inverse spectral problems}

In this section we apply Theorem 4.2 to inverse spectral problems for second-order operators on graphs. The first theorem gives a simple consequence of Theorem 4.2 while the second result uses the variational reformulation of (1.3), (1.4) to give a somewhat deeper result. 
Theorem 5.1. If $A, V_{p},\left\{\lambda_{j}\right\}$ and $\left\{\mu_{j}\right\}$ are as defined in Theorem 4.2, then for

$$
\sum_{j=0}^{\infty}\left(\mu_{j}-\lambda_{j}\right)
$$

convergent and $p$ of constant sign on $G$ we have that $p=0$ everywhere on $G$.

Proof. From Theorem 4.2, $\int_{G} p(x) \mathrm{d} x=0$, making $p=0$ almost everywhere and thus identically 0 , as $p \in \mathcal{C}^{2}(G)$.

The eigenvalue problem (1.3), (1.4), or equivalently the operator eigenvalue problem associated with $A$, has a variational or weak $\mathcal{H}^{1}(G)$ formulation and this was studied in $[\mathbf{1 6}]$. Without loss of generality, we assume the boundary conditions (1.4) to be of the form

$$
\begin{aligned}
\sum_{j=1}^{K}\left[\alpha_{i j} y_{j}(0)+\gamma_{i j} y_{j}\left(l_{j}\right)\right] & =0, \quad i=1, \ldots, J, \\
\sum_{j=1}^{K}\left[\alpha_{i j} y_{j}(0)+\beta_{i j} y_{j}^{\prime}(0)+\gamma_{i j} y_{j}\left(l_{j}\right)+\delta_{i j} y_{j}^{\prime}\left(l_{j}\right)\right] & =0, \quad i=J+1, \ldots, 2 K,
\end{aligned}
$$

where $y_{i}=\left.y\right|_{e_{i}}$ and $J$ is maximal, i.e. no conditions independent of $y_{j}^{\prime}(0)$ and $y_{j}^{\prime}\left(l_{j}\right)$ can be extracted by linear operations from (5.3).

Let $F(x, y)$ be the sesquilinear form given by

$$
F(x, y):=\int_{\partial G} f x \bar{y}+\int_{G}\left(x^{\prime} \bar{y}^{\prime}+x q \bar{y}\right) \mathrm{d} t,
$$

with domain

$$
\mathcal{D}(F)=\left\{y \in \mathcal{H}^{1}(G) \mid y \text { obeying }(5.2)\right\}
$$

where

$$
\int_{\partial G} y \mathrm{~d} \sigma:=\sum_{i=1}^{K}\left[y_{i}\left(l_{i}\right)-y_{i}(0)\right]=\int_{G} y^{\prime} \mathrm{d} t .
$$

Definition 5.2. The boundary conditions on $G$ are co-normal with respect to $l$ if there exists $f$ defined on $\partial G$ such that $x \in \mathcal{D}(F)$ obeys

$$
\int_{\partial G} f x \bar{y} \mathrm{~d} \sigma=\int_{\partial G} x^{\prime} \bar{y} \mathrm{~d} \sigma \quad \text { for all } y \in \mathcal{D}(F)
$$

if and only if $x$ obeys (5.3).

Most physically interesting boundary conditions on graphs are of co-normal type. In particular, Kirchhoff boundary conditions are co-normal. Observe that if node $\nu$ has Kirchhoff boundary conditions, then $f(x)=0$ for all $x \in \nu$ and this node contributes the domain conditions $y(x)=y(z)$ for all $x, z \in \nu$. 
If each node of $G$ has boundary conditions of Kirchhoff type, let $\Lambda_{K}$ denote the collection of nodes of $G$. Then

$$
\mathcal{D}(F)=\left\{y \in \mathcal{H}^{1}(G) \mid y(x)=y(z) \text { for all } x, z \in \nu, \text { for each } \nu \in \Lambda_{K}\right\},
$$

and $f$ is the constant 0 function on $\partial G$.

In [16] it was shown that if (5.2), (5.3) are co-normal boundary conditions with respect to $l$, then $u \in \mathcal{D}(F)$ satisfies $F(u, v)=\lambda(u, v)$ for all $v \in \mathcal{D}(F)$ if and only if $u \in H^{2}(G)$ and $u$ obeys (1.1), (5.2), (5.3).

Proof of Theorem 1.1. From Theorem 4.2 with $p=-q$, we obtain that $\int_{G} q(x) \mathrm{d} x=0$.

Let $\tilde{F}$ and $F$ denote the sesquilinear forms corresponding to the eigenvalue problems for $\tilde{A}$ and $A$, respectively, and let $\mathcal{D}(\tilde{F})=\mathcal{D}=\mathcal{D}(F)$ denote their domain as given in (5.5). Observe that

$$
\tilde{F}(x, y)=\int_{G}\left(x^{\prime} \bar{y}^{\prime}+x q \bar{y}\right) \mathrm{d} t
$$

and

$$
F(x, y)=\int_{G} x^{\prime} \bar{y}^{\prime} \mathrm{d} t
$$

Hence $F$ is positive definite on $\mathcal{D}$, making $\lambda_{0} \geqslant 0$. In addition, from the definition of $\mathcal{D}$ it is apparent that the constant 1 function $\mathbf{1}$ is in $\mathcal{D}$. Also $F(\mathbf{1}, \mathbf{1})=0$ and thus, from the variational formulation of the boundary-value problem in [16], 0 is the least eigenvalue of $A$ and has eigenfunction 1 . The hypotheses of the theorem now enable us to conclude that 0 is also the least eigenvalue of $\tilde{A}$, making $\tilde{F}$ positive semi-definite on $\mathcal{D}$. But the definition of $\tilde{F}$, along with the mean value of $q$ being 0 , gives

$$
\tilde{F}(\mathbf{1}, \mathbf{1})=\int_{G} q \mathrm{~d} t=0 .
$$

Hence $\mathbf{1}$ is an eigenfunction of $\tilde{A}$ with eigenvalue 0 , from [16].

In $[\mathbf{1 6}]$ it was shown that the eigenvalue problem for the operator $A$ and the boundaryvalue problem (1.3), (1.4) are equivalent. Consequently, $\mathbf{1}$ is an eigenfunction of (1.3), (1.4) for the eigenvalue 0 and so $q=-(\mathbf{1})^{\prime \prime}+q \cdot \mathbf{1}=0 \cdot \mathbf{1}=0$.

\section{Appendix}

If we impose Dirichlet boundary conditions at each node on the graph $G$, i.e. the condition that $y_{i}(0)=0=y_{i}\left(l_{i}\right)$ for all $i=1, \ldots, K$, then, from a boundary-value-problem perspective, the graph can be considered as a disconnected graph composed of the disjoint union of the edges $e_{i}$ each with Dirichlet boundary conditions at both ends.

Equation (1.3) with Dirichlet boundary conditions at each node has operator representation $\mathbb{A}$, where $\mathcal{D}(\mathbb{A})=\mathcal{H}^{2}(G) \cap \mathcal{H}_{\circ}^{1}(G)$ and has a particularly simple Green's function. Denote the iterates of this Green's function by $\gamma^{k}(x, y, \lambda), k \in \mathbb{N}$, i.e. the kernel of $\Gamma_{\lambda}^{k}:=(\mathbb{A}-\lambda I)^{-k}$. 
For $\rho>0$, denote $\sqrt{\lambda}=i \rho$.

Lemma 6.1. The iterated Green's function $\gamma^{k}(x, y, \lambda)$ of

$$
(l-\lambda) g=-\frac{\mathrm{d}^{2} g}{\mathrm{~d} x^{2}}+q g-\lambda g,
$$

with Dirichlet boundary conditions at each node, is given by

$$
\gamma^{k}(x, y, \lambda)= \begin{cases}0, & x \in e_{i}, y \in e_{j}, \text { where } i \neq j, \\ \gamma_{i}^{k}(x, y, \lambda), & x, y \in e_{i},\end{cases}
$$

where $\gamma_{i}^{k}(x, y, \lambda)$ is the iterated Green's function of $(l-\lambda)$ on the edge $e_{i}$ with Dirichlet boundary conditions at both ends.

Proof. If $f \in \mathcal{L}^{2}(G)$ and $x \in e_{i}$, then

$$
(\mathbb{A}-\lambda) \int_{G} \gamma(x, y, \lambda) f(y) \mathrm{d} y=(l-\lambda) \int_{0}^{l_{i}} \gamma_{i}(x, y, \lambda) f_{i}(y) \mathrm{d} y=f(x) .
$$

Also, for $x \in e_{i}$ and $f \in \mathcal{D}(\mathbb{A})$ we have

$$
\int_{G} \gamma(x, y, \lambda)(\mathbb{A}-\lambda) f(y) \mathrm{d} y=\int_{0}^{l_{i}} \gamma_{i}(x, y, \lambda)(l-\lambda) f_{i}(y) \mathrm{d} y=f(x),
$$

thus proving the claim for $k=1$.

Assuming the result for $k$ and letting $x \in e_{i}$, bootstrapping on the above case, we obtain that

$$
\gamma^{k+1}(x, y, \lambda)=\int_{G} \gamma(x, z, \lambda) \gamma^{k}(z, y, \lambda) \mathrm{d} z=\int_{0}^{l_{i}} \gamma_{i}(x, z, \lambda) \gamma^{k}(z, y, \lambda) \mathrm{d} z .
$$

But the hypothesis that the result holds for $k$ gives, for $z \in e_{i}$,

$$
\gamma^{k}(z, y, \lambda)= \begin{cases}0, & y \in e_{j}, \text { where } i \neq j \\ \gamma_{i}^{k}(z, y, \lambda), & y \in e_{i},\end{cases}
$$

and hence

$$
\gamma^{k+1}(x, y, \lambda)= \begin{cases}0, & y \in e_{j}, \text { where } i \neq j \\ \int_{0}^{l_{i}} \gamma_{i}(x, z, \lambda) \gamma_{i}^{k}(z, y, \lambda) \mathrm{d} z, & y \in e_{i},\end{cases}
$$

from which the result follows for $k+1$ upon noting that

$$
\gamma_{i}^{k+1}(x, y, \lambda)=\int_{0}^{l_{i}} \gamma_{i}(x, z, \lambda) \gamma_{i}^{k}(z, y, \lambda) \mathrm{d} z .
$$

The theorem now follows by induction. 
Lemma 6.2. For $q \in \mathcal{C}^{2(k-1)}(G)$ let $\gamma_{i}^{k}\left(x, y,-\rho^{2}\right)$ be as defined in Lemma 6.1. Then $\gamma_{i}^{k}$ has the following asymptotics approximations:

$$
\begin{aligned}
\gamma_{i}^{k}\left(x, y,-\rho^{2}\right) & =O\left(\frac{\mathrm{e}^{-\rho|x-y|}}{\rho^{k}}\right), \\
\frac{\partial \gamma_{i}^{k}\left(x, y,-\rho^{2}\right)}{\partial y} & =O\left(\frac{\mathrm{e}^{-\rho|x-y|}}{\rho^{k-1}}\right) \\
\gamma_{i}\left(x, y,-\rho^{2}\right) & =\frac{\mathrm{e}^{-\rho|x-y|}}{2 \rho}\left(1+O\left(\frac{1}{\rho}\right)\right), \\
\gamma_{i}^{2}\left(x, y,-\rho^{2}\right) & =\frac{\mathrm{e}^{-\rho|x-y|}}{4 \rho^{2}}\left[|x-y|\left(1+O\left(\frac{1}{\rho}\right)\right)+\frac{1}{\rho}\left(1+O\left(\frac{1}{\rho}\right)\right)\right],
\end{aligned}
$$

where (6.1) and (6.2) hold uniformly in $x$ and $y$ as $\rho \rightarrow+\infty$, while (6.3) and (6.4) hold uniformly for $(x, y)$ on compact subsets of $e_{i}^{\circ} \times e_{i}^{\circ}=\left(0, l_{i}\right) \times\left(0, l_{i}\right)$ as $\rho \rightarrow+\infty$.

Proof. By Lemma 6.1 we need only consider the case of $x, y \in e_{i}$. We proceed by induction on $k$.

$\boldsymbol{k}=1$. Let $S(x, \rho)$ be the solution $\left(l+\rho^{2}\right) S=0$ on $e_{i}^{\circ}=\left(0, l_{i}\right)$ having $S(0, \rho)=0$ and $S^{\prime}(0, \rho)=1$, then, from the appendix of $[\mathbf{2 6}]$,

$$
\begin{gathered}
S(x, \rho)=\frac{\sinh \rho x}{\rho}+O\left(\frac{\mathrm{e}^{\rho x}}{\rho^{2}}\right), \\
S^{\prime}(x, \rho)=\cosh \rho x+O\left(\frac{\mathrm{e}^{\rho x}}{\rho}\right) .
\end{gathered}
$$

Let $\sigma(x, \rho)$ be the solution of $\left(l+\rho^{2}\right) \sigma=0$ on $e_{i}$ with $\sigma\left(l_{i}, \rho\right)=0$ and $\sigma^{\prime}\left(l_{i}, \rho\right)=1$, then, again from the appendix of $[\mathbf{2 6}]$,

$$
\begin{gathered}
\sigma(x, \rho)=\frac{\sinh \rho\left(x-l_{i}\right)}{\rho}+O\left(\frac{\mathrm{e}^{l_{i}-x}}{\rho^{2}}\right), \\
\sigma^{\prime}(x, \rho)=\cosh \rho\left(x-l_{i}\right)+O\left(\frac{\mathrm{e}^{l_{i}-x}}{\rho}\right) .
\end{gathered}
$$

In (6.5)-(6.8) the approximations are uniform in $x$ as $\rho \rightarrow+\infty$.

The Green's function $\gamma_{i}$ can be explicitly expressed in terms of the solutions $S(x, \rho)$ and $\sigma(x, \rho)$ by

$$
\gamma_{i}\left(x, y,-\rho^{2}\right)= \begin{cases}\frac{S(x, \rho) \sigma(y, \rho)}{W[\sigma, S]}, & x \leqslant y, \\ \frac{\sigma(x, \rho) S(y, \rho)}{W[\sigma, S]}, & x \geqslant y\end{cases}
$$

(see $[\mathbf{1 4}]$ or $[\mathbf{3 0}$, pp. 35-37]). Here $W[\sigma, S](\rho)$ denotes the Wronskian of $\sigma(x, \rho)$ and $S(x, \rho)$, which has the argument $x$ omitted as it is independent of $x$ (see [14, p. 82]). It should be noted that $\gamma_{i}\left(x, y,-\rho^{2}\right)$ is a continuous function of $x$ and $y$ (see [30, p. 29]). 
Combining (6.5)-(6.8), direct computation gives

$$
W[\sigma, S](0, \rho)=-\frac{\mathrm{e}^{\rho l_{i}}}{2 \rho}\left(1+O\left(\frac{1}{\rho}\right)\right),
$$

as $\rho \rightarrow+\infty$.

Substituting (6.5), (6.7) and (6.10) into (6.9) gives

$$
\gamma_{i}\left(x, y,-\rho^{2}\right)=O\left(\frac{\mathrm{e}^{-\rho|y-x|}}{\rho}\right)
$$

uniformly in $x$ and $y$ for $\rho \rightarrow+\infty$. Also, uniformly for $(x, y)$ on compact subsets of $e_{i} \times e_{i}=\left(0, l_{i}\right) \times\left(0, l_{i}\right)$ as $\rho \rightarrow+\infty$ we have the more precise estimate

$$
\gamma_{i}\left(x, y,-\rho^{2}\right)=\frac{\mathrm{e}^{-\rho|x-y|}}{2 \rho}\left(1+O\left(\frac{1}{\rho}\right)\right) .
$$

We have thus established (6.1) for $k=1$ and (6.3).

Differentiating (6.9) with respect to $y$ yields

$$
\frac{\partial \gamma_{i}\left(x, y,-\rho^{2}\right)}{\partial y}= \begin{cases}\frac{S(x, \rho)}{W[\sigma, S](\rho)} \frac{\mathrm{d} \sigma(y, \rho)}{\mathrm{d} y}, & x<y, \\ \frac{\sigma(x, \rho)}{W[\sigma, S](\rho)} \frac{\mathrm{d} S(y, \rho)}{\mathrm{d} y}, & x>y,\end{cases}
$$

for all $x \neq y$ and $\rho>0$ large. Substituting the estimates (6.5)-(6.8) into (6.11) we obtain

$$
\frac{\partial \gamma_{i}\left(x, y,-\rho^{2}\right)}{\partial y}=O\left(\mathrm{e}^{-\rho|x-y|}\right)
$$

uniformly in $x$ and $y$ for $\rho \rightarrow+\infty$, thus proving (6.2) for $k=1$.

The induction step. For the remainder of the proof we assume that the lemma is true for $k$. We begin by considering (6.1) for $k+1$. From the definition of $\gamma_{i}^{k+1}$ it follows that

$$
\gamma_{i}^{k+1}\left(x, y,-\rho^{2}\right)=\int_{0}^{l_{i}} \gamma_{i}^{k}\left(x, z,-\rho^{2}\right) \gamma_{i}\left(z, y,-\rho^{2}\right) \mathrm{d} z .
$$

The induction hypothesis and (6.1) for the case of $k=1$ applied to (6.13), where the uniformity of the approximations is noted, gives

$$
\gamma_{i}^{k+1}\left(x, y,-\rho^{2}\right)=O\left(\int_{0}^{l_{i}} \frac{\mathrm{e}^{-\rho|x-z|}}{\rho^{k}} \frac{\mathrm{e}^{-\rho|y-z|}}{\rho} \mathrm{d} z\right) .
$$

Since $|x-z|+|z-y| \geqslant|y-x|$, the above equation yields

$$
\gamma_{i}^{k+1}\left(x, y,-\rho^{2}\right)=O\left(\frac{\mathrm{e}^{-\rho|x-y|}}{\rho^{k+1}}\right)
$$

uniformly in $x$ and $y$ as $\rho \rightarrow+\infty$, thereby proving (6.1). 
The proof of (6.2) follows from (6.1) and the case of (6.2) for $k=1$ since

$$
\begin{aligned}
\frac{\partial \gamma_{i}^{k+1}\left(x, y,-\rho^{2}\right)}{\partial y} & =\int_{0}^{l_{i}} \gamma_{i}^{k}\left(x, z,-\rho^{2}\right) \frac{\partial \gamma_{i}\left(z, y,-\rho^{2}\right)}{\partial y} \mathrm{~d} z \\
& =O\left(\int_{0}^{l_{i}} \frac{\mathrm{e}^{-\rho|x-z|}}{\rho^{k}} \mathrm{e}^{-\rho|y-z|} \mathrm{d} z\right),
\end{aligned}
$$

from which it follows, as in the case of the iterates of $\gamma_{i}$, that

$$
\frac{\partial \gamma_{i}^{k+1}\left(x, y,-\rho^{2}\right)}{\partial y}=O\left(\frac{\mathrm{e}^{-\rho|x-y|}}{\rho^{k}}\right)
$$

uniformly in $x$ and $y$ as $\rho \rightarrow+\infty$, thereby proving (6.2).

We now progress to the proof of (6.4). From (6.5), (6.7) and (6.9), observe that for $x \leqslant y$

$$
\begin{aligned}
\frac{1}{4} \rho^{2} \mathrm{e}^{2 \rho l_{i}} \gamma_{i}^{2}\left(x, y,-\rho^{2}\right) & \\
= & \frac{1}{4} \rho^{2} \mathrm{e}^{2 \rho l_{i}} \int_{0}^{l_{i}} \gamma_{i}\left(x, z,-\rho^{2}\right) \gamma_{i}\left(z, y,-\rho^{2}\right) \mathrm{d} z \\
= & \int_{0}^{x}\left(\sinh \rho\left(x-l_{i}\right) \sinh ^{2} \rho z \sinh \rho\left(y-l_{i}\right)+O\left(\frac{\mathrm{e}^{\rho\left(2 z+2 l_{i}-x-y\right)}}{\rho}\right)\right) \mathrm{d} z \\
& \quad+\int_{x}^{y}\left(\sinh \rho x \sinh \rho\left(z-l_{i}\right) \sinh \rho z \sinh \rho\left(y-l_{i}\right)+O\left(\frac{\mathrm{e}^{\rho\left(2 l_{i}+x-y\right)}}{\rho}\right)\right) \mathrm{d} z \\
& \quad+\int_{y}^{l_{i}}\left(\sinh \rho x \sinh ^{2} \rho\left(z-l_{i}\right) \sinh \rho y+O\left(\frac{\mathrm{e}^{\rho\left(2 l_{i}-2 z+x+y\right)}}{\rho}\right)\right) \mathrm{d} z \\
= & \sinh \rho\left(x-l_{i}\right) \sinh \rho\left(y-l_{i}\right)\left(-\frac{1}{2} x+\frac{\sinh 2 \rho x}{4 \rho}\right) \\
& +\sinh \rho x \sinh \rho\left(y-l_{i}\right)\left(-\frac{y-x}{2} \cosh \rho l_{i}+\frac{\sinh \rho\left(2 y-l_{i}\right)}{4 \rho}-\frac{\sinh \rho\left(2 x-l_{i}\right)}{4 \rho}\right) \\
& +\sinh \rho x \sinh \rho y\left(-\frac{l_{i}-y}{2}+\frac{\sinh 2 \rho\left(l_{i}-y\right)}{4 \rho}\right) \\
& +O\left(\frac{\mathrm{e}^{\rho\left(2 l_{i}+x-y\right)}}{\rho^{2}}+|x-y| \frac{\mathrm{e}^{\rho\left(2 l_{i}+x-y\right)}}{\rho}\right)
\end{aligned}
$$

uniformly in $x \leqslant y$ as $\rho \rightarrow+\infty$. If we relax the uniformity of the above estimates to uniformity in $(x, y)$ on compact subsets of $e_{i}^{\circ} \times e_{i}^{\circ}=\left(0, l_{i}\right) \times\left(0, l_{i}\right)$ and use the symmetry of $\gamma_{i}$, the above expression can be simplified to

$$
\gamma_{i}^{2}\left(x, y,-\rho^{2}\right)=\frac{\mathrm{e}^{-\rho|x-y|}}{4 \rho^{2}}\left[|x-y|\left(1+O\left(\frac{1}{\rho}\right)\right)+\frac{1}{\rho}\left(1+O\left(\frac{1}{\rho}\right)\right)\right],
$$

thereby proving (6.4).

The following corollaries follow from Lemma 6.2. 
Corollary 6.3. If $q \in \mathcal{C}^{(2 k-1)}(G)$ and $\gamma_{i}^{k}\left(x, y,-\rho^{2}\right)$ is as defined in Lemma 6.1, then

$$
\left|\frac{\partial^{j}}{\partial x^{j}} \gamma_{i}^{k}\left(x, y,-\rho^{2}\right)\right| \leqslant \frac{K}{\rho^{k-j}} \mathrm{e}^{-\rho|x-y|}
$$

uniformly in $x$ and $y$ as $\rho \rightarrow \infty$ for $0 \leqslant j<k, k \in \mathbb{N}$, and $K$ a constant.

Proof. By Lemma 6.1 we need only consider $x, y \in e_{i}$.

As in the proof of Lemma 3.1, let $b_{\rho, x}^{k}:=\left(A+\rho^{2}\right)^{k}$, where $x$ denotes the variable with respect to which $A$ is operating. For $f \in \mathcal{D}\left(\mathbb{A}^{j}\right) \supset \mathcal{C}_{0}^{\infty}(G)$ we have

$$
\Gamma_{i}^{k} b_{\rho}^{j} f=\Gamma_{i}^{k-j} f .
$$

Thus

$$
\int_{0}^{l_{i}} \gamma_{i}^{k}\left(x, y,-\rho^{2}\right) b_{\rho, y}^{j} \varphi(y) \mathrm{d} y=\int_{0}^{l_{i}} \gamma_{i}^{k-j}\left(x, y,-\rho^{2}\right) \varphi(y) \mathrm{d} y
$$

for $\varphi \in \mathcal{C}_{0}^{\infty}\left(e_{i}\right)$.

Since the boundary-value problem is formally self-adjoint with $q \in \mathcal{C}^{2(k-1)}(G)$ and since $\varphi \in \mathcal{C}_{0}^{\infty}\left(e_{i}\right)$ we obtain from the above equation

$$
\int_{0}^{l_{i}} b_{\rho, y}^{j} \gamma_{i}^{k}\left(x, y,-\rho^{2}\right) \varphi(y) \mathrm{d} y=\int_{0}^{l_{i}} \gamma_{i}^{k-j}\left(x, y,-\rho^{2}\right) \varphi(y) \mathrm{d} y
$$

Therefore, making

$$
b_{\rho, x}^{j} \gamma_{i}^{k}\left(x, y,-\rho^{2}\right)=\gamma_{i}^{k-j}(x, y) \quad \text { for } 0 \leqslant j<k
$$

and

$$
b_{\rho, x}^{k} \gamma_{i}^{k}\left(x, y,-\rho^{2}\right)=0 \quad \text { for } x \neq y .
$$

We now prove that the inequality

$$
\left|\frac{\partial^{j}}{\partial x^{j}} \gamma_{i}^{k}\left(x, y,-\rho^{2}\right)\right| \leqslant \frac{K_{k, j}}{\rho^{k-j}} \mathrm{e}^{-\rho|x-y|}
$$

holds for all $0 \leqslant j<2 k-1$, where $K_{k, j}$ is a constant.

Lemma 6.2 gives immediately that $(6.15)$ is true for $k=1,2$.

Suppose that $(6.15)$ is true for all $1,2, \ldots, k$, then by Lemma 6.2 we have that for $k$ replaced by $k+1$ and $j=0,1$, (6.15) holds, i.e.

$$
\gamma_{i}^{k+1}\left(x, y,-\rho^{2}\right) \mid \leqslant \frac{K_{k+1,0}}{\rho^{k+1}} \mathrm{e}^{-\rho|x-y|}
$$

and

$$
\left|\frac{\partial}{\partial x} \gamma_{i}^{2}\left(x, y,-\rho^{2}\right)\right| \leqslant \frac{K_{k+1,1}}{\rho^{k-1}} \mathrm{e}^{-\rho|x-y|}
$$

for $K_{k+1,0}, K_{k+1,1}$ constants. 
Now assume that

$$
\left|\frac{\partial^{j}}{\partial x^{j}} \gamma_{i}^{k+1}\left(x, y,-\rho^{2}\right)\right| \leqslant \frac{K_{k+1, j}}{\rho^{k+1-j}} \mathrm{e}^{-\rho|x-y|}
$$

is true for all $j=0,1, \ldots J$, where $1 \leqslant J \leqslant 2 k-2$.

Then, using Leibnitz's rule (see [1, p. 9]),

$$
\begin{aligned}
\left|\frac{\partial^{J+1}}{\partial x^{J+1}} \gamma_{i}^{k+1}\left(x, y,-\rho^{2}\right)\right| & =\left|\frac{\partial^{J-1}}{\partial x^{J-1}}\left(-b_{\rho, x}+q_{i}(x)+\rho^{2}\right) \gamma_{i}^{k+1}(x, y,-\rho 2)\right| \\
& =\left|\frac{\partial^{J-1}}{\partial x^{J-1}}\left[-\gamma_{i}^{k}\left(x, y,-\rho^{2}\right)+\left(q_{i}(x)+\rho^{2}\right) \gamma_{i}^{k+1}\left(x, y,-\rho^{2}\right)\right]\right| \\
& =\mid-\frac{\partial^{J-1}}{\partial x^{J-1}} \gamma_{i}^{k}\left(x, y,-\rho^{2}\right)+\rho^{2} \frac{\partial^{J-1}}{\partial x^{J-1}} \gamma_{i}^{k+1}\left(x, y,-\rho^{2}\right) \\
& \quad+\sum_{m \leqslant J-1}\left(\begin{array}{c}
m \\
J-1
\end{array}\right)\left(\frac{\partial^{m} q_{i}(x)}{\partial x^{m}}\right) \frac{\partial^{J-1-m}}{\partial x^{J-1-m}} \gamma_{i}^{k+1}\left(x, y,-\rho^{2}\right) \mid \\
& \leqslant \mathrm{e}^{-\rho|x-y|}\left(\frac{K_{k, J-1}}{\rho^{k-J+1}}+\frac{K_{k+1, J-1}}{\rho^{k-J}}+O\left(\frac{1}{\rho^{k-J+2}}\right)\right) \\
& =\mathrm{e}^{-\rho|x-y|} O\left(\frac{1}{\rho^{k-J}}\right) \\
& \leqslant K_{k+1, J+1} \frac{\mathrm{e}^{-\rho|x-y|}}{\rho^{k-J}}
\end{aligned}
$$

for $K_{k, J-1}, K_{k+1, J-1}$ and $K_{k+1, J+1}$ constants. Hence the lemma now follows.

Corollary 6.4. If $q \in \mathcal{C}^{3}(G)$ and $\gamma_{i}^{2}\left(x, y,-\rho^{2}\right)$ is as defined in Lemma 6.1, then

$$
\gamma_{i}^{2}\left(x, x,-\rho^{2}\right)=\frac{1}{4 \rho^{3}}\left[1+O\left(\frac{1}{\rho}\right)\right]
$$

uniformly for $x$ on compact subsets of $e_{i}^{\circ}=\left(0, l_{i}\right)$ as $\rho \rightarrow+\infty$.

Acknowledgements. The financial assistance of the National Research Foundation, grant TTK2007040500065, towards the research of S.C. is hereby acknowledged. B.A.W. was supported in part by the Centre for Applicable Analysis and Number Theory and by the South African National Research Foundation grant FA2007041200006.

\section{References}

1. S. Agmon, Lectures on elliptic boundary value problems (Princeton University Press, 1965).

2. F. Ali Menmeti, A characterization of a generalized $C^{\infty}$-notion on nets, Integ. Eqns Operat. Theory 9 (1986), 753-766.

3. F. V. AtKinson, Discrete and continuous boundary value problems (Academic Press, 1964). 
4. E. D. Belokolos, F. Gesztesy, K. A. Makarov and L. A. Sakhnovich, Matrixvalued generalizations of the theorems of Borg and Hochstadt, in Evolution equations (ed. G. R. Goldstein, R. Nagel and S. Romanelli), Lecture Notes in Pure and Applied Mathematics, Volume 234, pp. 1-34 (Dekker, New York, 2003).

5. J. Bochenek, On the inverse problem of the Sturm-Liouville type for a linear partial differential equation with constant coefficients of the second order, Ann. Polon. Math. 24 (1971), 331-341.

6. J. BocheneK, Global inverse problem of the Sturm-Liouville type for a linear elliptic partial differential equation of the second order, Ann. Polon. Math. 40 (1983), 271-281.

7. B. M. Brown and R. Weikard, A Borg-Levinson theorem for trees, Proc. R. Soc. Lond. A 461 (2005), 3231-3243.

8. R. CARlson, Adjoint and self-adjoint differential operators on graphs, Electron. J. Diff. Eqns 1998(6) (1998), 1-10.

9. R. CARlson, Inverse eigenvalue problems on directed graphs, Trans. Am. Math. Soc. 351 (1999), 4069-4088.

10. R. CARlson, Eigenvalue estimates and trace formulas for the matrix Hill's equation, $J$. Diff. Eqns 167 (2000), 211-244.

11. N. K. Chakravarty and S. K. Acharyya, On an extension of the theorem of V. A. Ambarzumyan, Proc. R. Soc. Edinb. A 110 (1988), 79-84.

12. S. Clark, F. Gesztesy, H. Holden and B. M.Levitan, Borg-type theorems for matrix-valued Schrödinger operators, J. Diff. Eqns 167 (2000), 181-210.

13. S. Clark, F. Gesztesy and W. Renger, Trace formulas and Borg-type theorems for matrix-valued Jacobi and Dirac finite difference operators, J. Diff. Eqns 219 (2005), 144182.

14. E. A. CODDINGTON AND N. LEVINSON, Theory of ordinary differential equations, pp. 87, 285 (McGraw-Hill, New York, 1955).

15. S. Currie AND B. A. WATson, Eigenvalue asymptotics for differential operators on graphs, J. Computat. Appl. Math. 182 (2005), 13-31.

16. S. CURrie And B. A. WATson, Dirichlet-Neumann bracketing for boundary value problems on graphs, Electron. J. Diff. Eqns 2005(93) (2005), 1-11.

17. B. Després, The Borg theorem for the vectorial Hill's equation, Inv. Probl. 11 (1995), 97-121.

18. L. GARDING, On the asymptotic distribution of the eigenvalues and eigenfunctions of elliptic differential operators, Math. Scand. 1 (1953), 237-255.

19. F. Gesztesy And B. Simon, The xi function, Acta Math. 176 (1996), 49-71.

20. F. Gesztesy, H. Holden, B. Simon and Z, ZhaO, Higher order trace relations for Schrödinger operators, Rev. Math. Phys. 7 (1995), 893-922.

21. R. C. Gilbert And V. A. Kramer, Trace formulas for a perturbed operator, Duke Math. J. 30 (1963), 275-296.

22. R. C. Gilbert and V. A. Kramer, Trace formulas for powers of Sturm-Liouville operators, Can. J. Math. 16 (1964), 412-422.

23. I. C. Gohberg And M. G. Krein, Introduction to the theory of linear non-self-adjoint operators (American Mathematical Society, Providence, RI, 1969).

24. B. Gutkin And U. Smilansky, Can one hear the shape of a graph, J. Phys. A 34 (2001), 6061-6068.

25. C. J. A. Halberg And V. A. Kramer, A generalization of the trace concept, Duke Math. J. 27 (1960), 607-617.

26. H. Hochstadt, On inverse problems associated with Sturm-Liouville operators, J. Diff. Eqns 17 (1975), 220-235.

27. V. Hutson And J. S. Pym, Applications of functional analysis and operator theory (Academic Press, 1980). 
28. V. A. JAVRJAN, On the regularized trace of the difference between two singular SturmLiouville operators, Dokl. Akad. Nauk SSSR 169 (1966), 888-891.

29. P. Kurasov, Graph Laplacians and topology, preprint (Lund Institute of Technology, Lund University, 2006).

30. M. A. NAIMARK, Linear differential operators, Parts I and II (Frederick Ungar, New York, 1967, 1968).

31. W. Pielichowski, Asymptotic behaviour of iterated Green's functions of a linear elliptic differential operator, Univ. Iagel. Acta Math. 27 (1988), 229-238.

32. W. Pielichowski, An inverse problem for linear elliptic differential operators, Univ. Iagel. Acta Math. 27 (1988), 239-246.

33. V. Pivovarchik, Inverse problems for the Sturm-Liouville equation on a simple graph, SIAM J. Math. Analysis 32 (2000), 801-819.

34. V. Pivovarchik, Ambarzumian's theorem for a Sturm-Liouville boundary value on a star shaped graph, Funct. Analysis Applic. 39 (2005), 148-151.

35. V. Pivovarchik, Inverse problem for the Sturm-Liouville equation on a star shaped graph, Math. Nachr. 280 (2007), 1595-1619.

36. Yu. V. Pokornyi and V. L. Pryadiev, Some problems of the qualitative SturmLiouville theory on a spatial network, Russ. Math. Surv. 59(3) (2004), 515-552.

37. W. T. REID, Ordinary differential equations (Wiley, 1971).

38. J. VON BELOW, A characteristic equation associated to an eigenvalue problem on $c^{2}$ networks, Linear Alg. Applic. 71 (1985), 309-325.

39. J. Weidmann, Linear operators in Hilbert space (Springer, 1980).

40. V. YuRKo, Inverse spectral problems for Sturm-Liouville operators on graphs, Inv. Probl. 21 (2005), 1075-1086.

41. V. YuRKo, On recovering Sturm-Liouville operators on graphs, Math. Notes 79 (2006), $572-582$. 